\title{
Immortal time bias in the association of n-3 fatty acid supplementation and atrial fibrillation
}

\begin{abstract}
Alvaro Alonso
Received: 16 November 2008 / Accepted: 7 January 2009/Published online: 7 February 2009

(C) Springer-Verlag 2009
\end{abstract}

Keywords Pharmacoepidemiology Immortal time bias

Macchia et al. have employed a computerized health database to assess the effect of n-3 polyunsaturated acids (PUFA) supplements in the prevention of atrial fibrillation (AF) and reduction of mortality rates in patients who have suffered a myocardial infarction [1]. After adjusting for potential confounders, they found that use of n-3 PUFAs was associated with reductions of $81 \%$ and $85 \%$ in the rates of AF and total mortality, respectively [1]. The use of large computerized databases including a variety of clinical information is, certainly, a useful and efficient approach to estimate the effect of interventions in large populations and in real-life clinical situations. Propensity scores, additionally, if properly applied, can help to control for measured confounding.

However, the results presented by Macchia et al. could be affected by what pharmacoepidemiologists usually call “immortal time bias" [2]. In Macchia's study, individuals were considered exposed if they were prescribed n-3 PUFA at some point before or after the beginning of follow-up. This definition of exposure is problematic since exposed individuals who received n-3 PUFA at some time during

A. Alonso $(\bowtie)$

Division of Epidemiology and Community Health,

School of Public Health, University of Minnesota,

1300 S 2nd St., Suite 300,

Minneapolis, MN 55454, USA

e-mail: aalogut@alumni.unav.es the follow-up were, by definition, "immortal" during the period prior to the prescription, that is, they could not develop the endpoint (AF, mortality) during that time. In other words, to be classified as exposed, an individual needs to remain event free until the start of the exposure. If they had developed the endpoint, that endpoint would have been counted among the unexposed group. As a consequence, the person-time of follow-up among the exposed includes time in which the individuals cannot develop the outcome and, therefore, the incidence rates in that group are an underestimation of the true rates and the hazard ratios are downwardly biased.

A solution to this problem would be to conduct a Cox proportional hazards model with time-dependent exposures [3]. This method appropriately allocates exposed and unexposed person-time, removing this type of bias. I suggest the authors repeat their analysis using this methodology to provide a more valid estimate of the association between n-3 PUFA use and mortality and risk of AF after a myocardial infarction.

\section{References}

1. Macchia A, Monte S, Pellegrini F et al (2008) Omega-3 fatty acid supplementation reduces one-year risk of atrial fibrillation in patients hospitalized with myocardial infarction. Eur J Clin Pharmacol 64:627-634

2. Suissa S (2008) Immortal time bias in pharmacoepidemiology. Am J Epidemiol 167:492-499

3. Zhou Z, Rahme E, Abrahamowicz M et al (2005) Survival bias associated with time-to-treatment initiation in drug effectiveness evaluation: a comparison of methods. Am J Epidemiol 162:10161023 